\title{
Los insectos (Insecta) del Mesozoico chileno
}

\author{
The insects (Insecta) \\ from the chilean Mesozoic
}

Vicente Pérez ${ }^{1} \&$ Ernesto Pérez ${ }^{2}$

La Clase Insecta, con aproximadamente 1,8 millones de especies descritas (nominadas) y con 10-20 millones estimadas, es la más grande del Phylum Arthropoda (Gordh \& Headrick, 2011), y cada día se describen más especies. Tanto así que en los últimos años han surgido revistas consideradas de muy alto nivel, y dedicadas exclusivamente al quehacer taxonómico, como Phytotaxa, Zookeys y Zootaxa; ésta última con una impresionante periodicidad, ya que publica un número diario desde el año 2009 (Zhang, 2009).

A la fecha, no existen catálogos o listas de especies de insectos recientes o fósiles, actualizadas, y posiblemente no se logre nunca una recopilación exhaustiva de ellos.

Sabrosky (1952) ofrece las primeras cifras de especies actuales que podemos utilizar en relación con esta nota: 625.000 a 1.500 .000 (pág. 1), entre 625.000 y 1.115 .000 (pág.2), descritas hasta fines de 1948, Coleoptera 277.000, Hemiptera (en la acepción actual) 55.000 y Odonata 4.870 especies.

Dichas cifras no están muy alejadas de las que se aceptan generalmente para las especies vivientes en la actualidad para estos tres Órdenes. Coleoptera: 350.000 (Southwood, 1978), 350.000 (Liebherr \& McHugh, 2003), 300.000400.000 (Stork, 2003); Hemiptera (verdaderas chinches, i.e. Heteroptera): 40.000 (Leftwich, 1976), 55.000 (Resh \& Cardé, 2003), 42.347 Henry (2009); Odonata: 5471 (Tennessen, 2003).

Carpenter (1952) registra unas 12.000 especies de insectos fósiles. Aunque no sean tan abundantes como las de otros grupos de animales, el registro fósil de los insectos se extiende hacia el pasado hasta 400 millones de años, haciendo de este taxón uno de los más antiguos de los animales terrestres (Grimaldi, 2003).

El objetivo de esta contribución es dar a conocer el estado actual del conocimiento de los insectos del Mesozoico de Chile, destacando los grupos taxonómicos al que pertenecen y en qué tiempo habitaron nuestro territorio. La importancia del estudio de los insectos fósiles radica en que son testigos de una diversidad biológica antigua, permiten establecer líneas de filogenia, dan ejemplos de extinciones biológicas y aportan datos sobre paleoclimatología y paleobiogeografía (Martínez-Delclòs, 1996b).

La Era Mesozoica (245-65 millones de años antes del presente), es el intervalo de la Escala de Tiempo Geológico que se caracterizó por el origen, evolución, radiación adaptativa y extinción de los dinosaurios y reptiles voladores. Durante esta Era se originaron y persistieron las aves, hubo una importante propagación de gimnospermas (e.g. ginkgos, cicadoideas, cicadas y coníferas) y aparición de angiospermas.

El registro estratigráfico divide a la Era Mesozoica en tres Períodos geológicos: Triásico (245-208 MAAP), Jurásico (208-146 MAAP, Edad de las Cicadas) y Cretácico (146-65 MAAP) (Gordh

\footnotetext{
1 Laboratorio de Entomología, Instituto de la Patagonia, Universidad de Magallanes. vicente.perez@umag.cl

2 Servicio Nacional de Geología y Minería, Santiago († 19 de diciembre de 2013).
} 
\& Headrick, 2011).

La información registrada de insectos fósiles es muy escasa, aislada y discontinua, tanto en la distribución espacial como en el tiempo, si se la compara con la de otros grupos, por ejemplo, la de los vertebrados (Osuna, 1995).

En general, los yacimientos paleoentomológicos no son abundantes, ya que después que los insectos caen al agua, deben romper la tensión superficial de ella, llegar a un fondo acuático anóxico y una sedimentación rápida para evitar que sus cuerpos sean atacados por microorganismos necrófagos que digieren rápidamente la quitina y proteína, principales componentes del exoesqueleto de estos artrópodos (Peñalver et al. 1999; Martínez-Delclòs, 1996a; Martínez-Duclòs, 1996a; Carpenter \& Burnham, 1985). Los insectos fósiles se encuentran, por lo general, en sedimentitas continentales, lacustres o fluviales (excepcionalmente en yacimientos marinos), debido a lo cual la fosilización resulta bastante excepcional, por los ritmos poco constantes de la sedimentación, por la erosión y presencia de hiatos. Sin embargo, el exoesqueleto quitinoso de los insectos ha favorecido su fosilización, aunque casi siempre corresponde a partes de él como los élitros.

Los hallazgos de insectos del Mesozoico en Chile son escasos, refiriéndose exclusivamente a pterigotos, i.e. especies filogenéticamente aladas, y pertenecientes a los órdenes Coleoptera (Ademosyne hexacosta, Ademosyne sp., Ischichucasyne santajuanensis, Dorotheus guidensis), Hemiptera (Bandelnielsenia chilena) y Odonata (trazas de oviposición).

Las edades inferidas corresponden al Triásico Superior y Cretácico Superior. Se han encontrado principalmente en estratos de las formaciones Santa Juana (Región de Biobío), La Ternera (Región de Atacama) y Dorotea (Región de Magallanes).

Contrasta el pequeño número de especies de Chile con las de Argentina, Brasil, Australia, pero se destacan sus afinidades gondwánicas (Gallego et al. 2001; Stipanicic \& Marsicano, 2002).

Para la historia de la Paleontología en Chile podemos consignar que el primer insecto fósil (un élitro de coleóptero bien preservado) fue encontrado en Chile por el geólogo Giovanni Cecioni y fue dado a conocer en una nota publicada en el Boletín de la Universidad de Chile por el entomólogo Guillermo Kuschel (1959). El citado autor (1959) publicó, posteriormente, un artículo en Investigaciones Zoológicas Chilenas, en el que lo denominó Dorotheus guidensis gen. et sp. nov. La nueva especie pertenece al Orden Coleoptera, Familia Curculionidae, y fue hallada en el cerro Guido, norte de Última Esperanza, Región de Magallanes, en estratos de limolita azulada clara y fisible del contacto entre las formaciones Dorotea y Cerro Rincón Negro. Habría medido unos 7,0 mm de largo y habitado las estepas. La flora asociada al insecto (principalmente helechos) es considerada de edad cretácica superior, Piso Maastrichtiano (Kuschel, 1959). El holotipo de la nueva especie, No. J.C. 1693, está depositado en el Museo Nacional de Historia Natural de Santiago de Chile.

Fuenzalida (1937) y Cecioni \& Westermann (1968) señalaron la presencia de crustáceos e insectos indeterminados, de edad triásica, en el área de Los Molles, en la Región de Valparaíso.

Cabe destacar aquí los siguientes aspectos referidos al estudio de los insectos fósiles en Chile:

a) El avance del conocimiento sobre los insectos fósiles de Chile se ha visto favorecido, en la última década, por proyectos multinacionales relacionados principalmente con el estudio de conchostracos del Gondwana, de edad triásica superior, que han proporcionado registros de insectos en Argentina, Brasil, Chile y Uruguay.

b) Han participado paleoentomólogos y geólogos paleontólogos, como Oscar Florencio Gallegos, que ha actuado como líder, Silvia Gnaedinger, Oscar Kirsten, Susana Giovanelli (Universidad Nacional del Nordeste, Argentina); Rafael Goia Martins-Neto (Universidad Guarulhos, Brasil); Ricardo Néstor Melchor (Universidad Nacional de la Pampa), K. Adami Rodrigues (Universidad Federal do Acre, Brasil) y Sven N. Nielsen (Universidad de Kiel, Alemania).

c) Ejemplares recolectados en Chile dieron lugar a la descripción de especies nuevas y proposición de géneros nuevos.

d) Estos proyectos multinacionales han permitido comprobar que algunos taxones de insectos son especialmente útiles como indicadores biocronoestratigráficos, para 
establecer equivalencias locales y regionales, a nivel sudamericano, como es el caso del género Ademosyne (Gallego y Martins-Neto, 2005), de amplia distribución en el ámbito gondwánico.

Evidencias de la relación insecto-planta:

a) Galerías producidas por insectos en hojas de Taeniopteris del Triásico Superior (Gallego et al. 2003). Taeniopteris Brongniart, 1828, es un género que se encuentra en el centro de las cicadófitas, cuya sistemática aún no se ha establecido bien (Vakhrameev, 1991) y del cual se han descrito numerosas especies (Gaedinger \& Herbst, 2013).

b) Trazas de oviposición de insectos (Odonata), como impresiones en hojas de Heidiphyllum elongatum Retallack (Gnaedinger et al. 2007), de edad triásica superior (Formación La Ternera, sur de Copiapó, Región de Atacama).

Tabla 1. Sistemática de las especies del Mesozoico chileno

Phylum Arthropoda von Siebold, 1845 Clase Insecta Linnaeus, 1758

(Devónico a Reciente)

Orden Coleoptera Linnaeus, 1758

(Pérmico Inferior a Reciente)

Familia Permosynidae Tillyard, 1924

Género Ademosyne Handlirsch, 1906

Como conclusión, se puede decir que la escasez de los registros fósiles de insectos en Chile se debe en gran medida a la natural dificultad de preservación del grupo. Sin embargo, podrían incrementarse los hallazgos mediante recolecciones sistemáticas en estratos continentales de gran desarrollo en Chile como son las formaciones del Triásico Superior, que tienen la particularidad favorable y única en Sudamérica de contar con intercalaciones marinas, con invertebrados diagnósticos, que permiten una datación segura de las capas portadoras de insectos, vegetales y crustáceos.

\section{Ademosyne hexacostata Martins-Neto y Gallego, 2006}

Localidad y edad: Río Gualo, La Rioja, Argentina; Río Biobío, Región del Biobío. Triásico Superior temprano (Martins-Neto, Gallego,
Mancuso, 2006).

Depósito: (material argentino) Museo de Ciencias Naturales, Universidad Nacional de la Rioja. Holotipo PULR-I 305.

Ademosyne sp. Gallego y

Martins-Neto, 2005

Localidad y edad. Santa Juana, Región del Biobío. Triásico Superior (Gallego, Martins-Neto, Nielsen, 2005).

Depósito: Museo Nacional de Historia Natural (Chile), SGO.PI.6139 (2A), y (2B).

Género Ischichucasyne Martins-Neto y Gallego, 2005

Ischichucasyne santajuanaensis Martins-Neto y Gallego, 2005

Localidad y edad: Santa Juana, Región del Biobío. Triásico Superior.

Depósito: Museo Nacional de Historia Natural (Chile). Holotipo SGO.PI.6140.

Familia Curculionidae Latreille, 1802

Género Dorotheus Kuschel, 1959

Dorotheus guidensis Kuschel, 1959

Localidad y edad: Cerro Guido, Última Esperanza, Región de Magallanes. Cretácico Superior (Maastrichtiano).

Depósito: Museo Nacional de Historia Natural (Chile). Holotipo No. J.C. 1693.

Familia Cerambycidae Latreille, 1802

Género Dorotheus Kuschel, 1959

Australocerambyx chilensis Peña, 1959

Trazas indet.

Localidad y edad: norte del Estrecho de Magallanes, estepa patagónica; lugar no precisado. Edad desconocida.

Depósito: Museo Nacional de Historia Natural (Chile): muestra 1 (tipo) y 4, 5, 7, 8; Museo de la Patagonia (Punta Arenas): muestras 2 y 6; Colección de Luis E. Peña: muestra 3.

Nota: se registra esta especie, de edad 
desconocida, previendo el hallazgo de nuevos antecedentes.

Orden Hemiptera Linnaeus, 1758 (Pérmico Inferior a Reciente)

Suborden Auchenorryncha Duméril, 1806

Familia Dysmorphoptilidae Handlirsch, 1906

Género Bandelnielsenia Martins-Neto y Gallego, 2003

\section{Bandelnielsenia chilena Martins-Neto y Gallego, 2003}

Localidad y edad: sur de Santa Juana, sureste de Concepción, Región del Biobío. Triásico Superior.

Depósito: Museo Nacional de Historia Natural (Chile). Holotipo SGO.PI.5989.

Nota: "Auchenorhyncha surgió en el Paleozoico, apareciendo primero en el registro fósil del Pérmico Inferior (hace 280 millones de años)" (Dietrich, 2003).

Orden Odonata Fabricius, 1793

(Pérmico Inferior a Reciente)

Taxones infraorden indet. Cicatrices de oviposición (Gnaedinger, et al. 2007)

Localidad y edad: localidad no especificada. Formación La Ternera. Región de Atacama. Triásico Superior.

\section{LITERATURA CITADA}

Carpenter, F.M. (1963). Presentación de los Insectos. Insectos fósiles, pp. 16-22. En Centro Regional de Ayuda Técnica. Agencia para el Desarrollo Internacional (A.I.D.). Insectos. Plagas de la Agricultura y sistemas para combatirlos. México. 1154 pp. (Traducción al español de The Yearbook of Agriculture. Insects. United States Department of Agriculture. Washington, D.C.)

Carpenter, F.M. \& Burnham, L. (1985). The geological record of insects. Annual Review of Earth and Planetary Sciences, 13, 297314.

Cecioni, G. \& Westermann, G.E.G. (1968).
The Triassic/Jurassic marine transition of coastal central Chile. Pacific Geology, 1, 41-75.

Dietrich, C.H. (2003). Auchenorhyncha (Cicadas, Spitlebugs, Leafhoppers, Treehoppers, and Planthoppers). In V.H. Resh \& R. Cardé (Eds.) Encyclopedia of Insects (pp. 66-74). Academic Press.

Fuenzalida, H. (1937). El Rético en la costa de Chile Central. Ministerio de Fomento, Departamento de Minas y Petróleo, 6(65), 739-747.

Gallego, O.F. \& Martins-Neto, R.G. (2006). Propuesta preliminar sobre la distribución estratigráfica de las faunas triásicas de insectos y conchostracos de Argentina, Chile y sur de Brasil. Universidad Nacional del Nordeste (Argentina), Comunicaciones Científicas y Tecnológicas. 4 pp.

Gallego, O.F., Martins-Neto, R.G. \& Carmona, M.J. (2001). Nuevos registros de artrópodos (Insecta y Conchostraca) en el Triásico de la Argentina: comentarios sobre su afinidad con faunas de Laurasia y Gondwana. Universidad Nacional del Nordeste (Argentina), Comunicaciones Científicas y Tecnológicas. 4 pp.

Gallego, O.F. \& Martins-Neto, R.G. (2005). Insect fauna. In O.F. Gallego, R.G. Martins-Neto \& S.N. Nielsen. Conchostracans and insects from the Upper Triassic of the Biobío river ('Santa Juana Formation'), south-central Chile (pp. 300-303). Revista Geológica de Chile, 32(2), 293-311.

Gallego, O.F., Gnaedinger, S., Kirsten, O. \& Giovanelli, S. (2003). Primera cita de trazas fósiles de insectos en hojas del Pérmico de Uruguay y Triásico de Chile. Universidad Nacional del Nordeste, Comunicaciones Científicas y Tecnológicas, 4 pp.

Gnaedinger, S., Adami Rodrigues, K. \& Gallego, O.F. (2007). Evidencias de trazas de oviposición de insectos (Odonata) en hojas del Triásico Superior de Chile. Ameghiniana, 44(4). Suplemento. Resúmenes: 94-R-95R.

Gnaedinger, S. \& Herbst, R. (2013). Pteridophylla del Triásico del Norte Chico de Chile I. El género Taeniopteris Brongniart. Ameghiniana [S.1.], 41(1), 91-110. 
Gordh, G. \& Headrick, D. (2011). A Dictionary of Entomology. (2 $2^{\text {nd }}$ Edt.) Printed and bound in the UK by CPI Anthony Rowe, Chippenham.

Grimaldi, D. 2003. El registro fossil. In V.H. Resh \& R.T. Cardé (Eds.). Encyclopedia of Insects (pp. 455-463). Academic Press.

Henry, T.J. (2009). Biodiversity of Heteroptera. In R. Foottit, \& P. Adler (Eds.) .Insect Biodiversity: Science and Society. (1 ${ }^{\text {st }}$ Edition) pp. 223-263). Blackwell Publishing.

Kuschel, P.G. (1959). El primer insecto fósil encontrado en Chile. Universidad de Chile, Boletín, (5), 39.

Kuschel, P.G. (1959). Un curculiónido del Cretácico Superior. Primer insecto fósil de Chile. Investigaciones Zoológicas Chilenas, 5, 49-54. Santiago.

Leftwich, A.W. (1976). A Dictionary of Entomology. Constable. London.

Liebherr, J.K. \& McHugh, J.V. (2003). Coleoptera (Beetles, Weevils, Fireflies). In V.H. Resh \& R.T. Cardé (Eds.). Encyclopedia of Insects (pp. 209-230). Academic Press.

Martínez-Delclòs, X. (1996a). El registro fósil de los insectos. Boletín de la Asociación Española de Entomología, 20(1-2),9-30.

Martínez-Delclòs, X. (1996b). Origen y diversificación de los insectos. Su registro fósil. Bol. SEA, 16, Paleontología, pp. 125-138.

Martins-Neto, R.G. \& Gallego, O. F. (2003). [Bandelnielsenia $\mathrm{n}$. gen \& B. chilena $\mathrm{n}$. sp.], pp. In R. G., Martins-Neto, O.F. Gallego \& R.N. Melchor. The Triassic insect fauna from South America (Argentina, Brazil and Chile): a checklist (except Blattoptera and Coleoptera) and descriptions of new taxa. Acta zoologica cracoviensia, 46 (suppl. Fossil Insects): 229-256.

Martins-Neto, R.G. \& Gallego, O.F. (2006). Systematic Paleontology. In R.G. Martins-Neto, O.F. Gallego, \& A.C. Mancuso. The Triassic insect fauna from Argentina. Coleoptera from Los Rastros Formation (Bermejo Basin), La Rioja Province (pp. 592-601). Ameghiniana, 43(3):591-609.

Osuna, E. (1995). Morfología del Exoesqueleto de los Insectos. Volumen I. Origen y evolución, el exoesqueleto. Universidad Central de Venezuela. Consejo de Desarrollo Científico y Humanístico.

Peñalver, E.X. Martínez-Delclòs \& Arillo, A. (1999). Yacimientos con insectos fósiles en España. Revista Española de Paleontología, 14(2), 231-245.

Resh, V.H \& Cardé, R.T. (2003). Insecta, Overview. In V.H. Resh \& R.T. Cardé, Encyclopedia of Insects (pp.564-566). Academic Press.

Sabrosky, C.W. (1963). Presentación de los insectos. ¿Cuántos insectos existen? In Centro Regional de Ayuda Técnica. Agencia para el Desarrollo Internacional (A.I.D.). Insectos. Plagas de la Agricultura y sistemas para combatirlos (pp. 1-8). México. Traducción al español de The Yearbook of Agriculture. Insects. 1952. United States Department of Agriculture. Washington D.C.

Southwood, T.R.E. (1978). The components of diversity. In L.A. Mound \& N. Waloff (Eds.). Diversity of Insect Faunas. Symposia of the Royal Entomological Society of London. 9Blackwell Scientific Publications, Oxford.

Stipanicic, P.N. \& Marsicano, C.A. (2002). Léxico Estratigráfico de la Argentina. Volumen VIII. Triásico. Asociación Geológica Argentina. Serie " $B$ " (Didáctica y Complementaria). In O. Gallego \& R.G. Martins-Neto (Eds). Propuesta preliminar sobre la distribución estrtigráfica de las faunas triásicas de insectos y conchostracos de Argentina, Chile y sur de Brasil. Universidad Nacional del Nordeste. 26, 370 pp. Comunicaciones Científicas y Tecnológicas 2006. Resumen B-009. 4 pp. Stork, N.E. (2003). Biodiversity. In V.H. Resh \& R.T. Cardé (Eds.). Encyclopedia of Insects (pp. 89-91). Academic Press.

Tennessnen, K.J. (2003). Odonata (Dragonflies, Damselflies). In V.H. Resh \& R.T. Cardé (Eds.). Encyclopedia of Insects (pp. 814823). Academic Press.

Vakhrameev, V.A. (1991). Jurassic and Cretaceous Floras and Climates of the Earth. Cambridge University Press.

Zhang, Z. (2009). A daily journal of taxonomy for rapid species discovery. Zootaxa, 2120, $1-2$. 
